\title{
Primer programa institucional de uso racional de antibióticos en Gipuzkoa. Evaluación de los años 1999-2004
}

First program on rational use of antibiotics in Gipuzkoa. Assessment of 1999-2004 period Miguel Ángel GASTELURRUTIA, Belen LARRAÑAGA, Begoña ORTEGA.

\begin{abstract}
RESUMEN *
Objetivo: En el presente trabajo se analizan los resultados, hasta el año 2004, del Programa de Uso Racional de Antibióticos que viene realizándose en Gipuzkoa desde el año 1999 y en el que participan de forma voluntaria casi la mitad de las farmacias. Método: La recogida de datos se realiza en las farmacias y consiste en anotar todos los antibióticos solicitados y dispensados sin receta durante una semana cada trimestre, junto a otra información referida a la actuación del farmacéutico. Finalmente se remiten los datos al COF de Gipuzkoa.

Resultados: Durante este periodo se ha producido una disminución considerable de la solicitud de antibióticos sin receta, hasta el 3,6\% sobre el total de solicitudes de antibióticos. Por su parte, la dispensación de antibióticos sin receta disminuyó de manera significativa, hasta alcanzar el 1,6\% del total de dispensaciones de antibióticos. Esto supone que todavía se siguen dispensando antibióticos sin receta.

Conclusiones: Este programa ha conseguido disminuir tanto la solicitud como la dispensación de antibióticos sin receta. Es necesario seguir trabajando en este tipo de programas para reforzar la actuación profesional del farmacéutico, mediante la implicación más activa de las instituciones, la utilización de unas guías de actuación y la posible aplicación de incentivos negativos.
\end{abstract}

Palabras clave: Uso racional. Antibioticos.

\begin{abstract}
Objective: Results of the first program on rational use of antibiotics in Gipuzkoa, until the year 2004, are analyzed. This program was set up in 1999 and almost half of the pharmacies in the province have participated in it in a voluntary way.

Method: Data were gathered within the pharmacies during one week every three months, and by writing up all the antibiotics, both demanded and dispensed without corresponding prescription, and also some information related to the activity of the pharmacists in the dispensing process. All data were sent to the Gipuzkoa Professional Association.

Results: antibiotics' demand without prescription diminished to reach a $3.6 \%$ out of the overall antibiotics demand. On the other hand, antibiotics dispensing without the prescription fell until the $1.6 \%$ out of the overall antibiotics dispensed. These figures mean that a few antibiotics keep being dispensed without the corresponding prescription. Conclusions: This program achieved a reduction on both the demand and the dispensing of antibiotics without the corresponding prescription. It's important to keep working on these kind of programs to reinforce the professional role of pharmacists by a more active participation of related organizations, the use of protocols and guidelines, and the possible use of negative incentives.
\end{abstract}

Palabras clave: Rational use of drugs. Antibiotics.

(Español)

\section{INTRODUCCIÓN}

En 1999 el COF de Gipuzkoa, consciente de la gravedad de las consecuencias a nivel de salud pública que un uso inadecuado de los antibióticos podría producir, y siendo los farmacéuticos comunitarios miembros del equipo sanitario, decidió hacer un esfuerzo para mejorar la educación sanitaria tanto a profesionales, en nuestro caso a farmacéuticos, como a la población general. ${ }^{1}$

Miguel Ángel GASTELURRUTIA. Doctor en Farmacia. Farmacéutico comunitario en San Sebastián. Grupo de Investigación en Atención Farmacéutica de la Universidad de Granada (España).

Belen LARRAÑAGA. Farmacéutica. Colegio Oficial de Farmacéuticos de Gipuzkoa (España).

Begoña ORTEGA. Farmacéutica. Colegio Oficial de Farmacéuticos de Gipuzkoa (España).

Junto a ese objetivo general, se pretendía también conocer con la mayor exactitud posible la realidad de la dispensación, de la prescripción oral y de la 
automedicación en relación a la antibioterapia en nuestro medio. Tal como definimos en nuestra anterior publicación, entendemos por Prescripción oral como la recomendación de un medicamento que realiza el médico u odonto-estomatólogo sin utilizar el documento oficial de receta médica (p.ej. por teléfono) y como Automedicación, la solicitud de un medicamento sin prescripción médica, sea cual sea su origen, excluyendo la "prescripción oral".

Por último, y dado que el papel del farmacéutico es fundamentalmente garantizar el uso racional de los medicamentos, se consideró necesario mejorar la calidad del acto de dispensación mediante el mayor rigor posible en la exigencia de la receta médica.

Para alcanzar esos objetivos, se puso en marcha un Programa que comenzó en abril de 1999 y que sigue vigente en la actualidad. Los resultados de la evaluación de los datos de la primera fase 19992000 ya se han publicado.

En este trabajo se presenta la evaluación de los resultados obtenidos desde el comienzo del programa (abril de 1999) hasta octubre de 2004. Este periodo ha sido dividido en cuatro fases, cada una de ellas compuesta a su vez por cuatro semanas de recogida de datos. La primera fase se corresponde con los meses de Abril'99, Julio'99, Octubre'99 y Enero'00. La segunda fase con Julio'00, Octubre'00, Enero'01 y Abril'01. La tercera fase: Julio'02, Octubre'02, Enero'03 y Abril'03; y la cuarta fase: Febrero'04, Abril'04, Julio'04 y Octubre'04.

El propósito de esta publicación es conocer la evolución y características de la "solicitud de antibióticos" durante el periodo de estudio, relacionando la actuación del farmacéutico y la solicitud de antibióticos sin receta. Además, se pretende estudiar de la misma manera la evolución y características de la "dispensación de antibióticos" durante el periodo de estudio. En este sentido se analizó: la dispensación sin receta, la evolución de la dispensación sin receta en función del origen de su solicitud (prescripción oral o automedicación), la actuación del farmacéutico ante esta solicitud sin receta y la relación entre la dispensación sin receta con las variables predictoras del estudio. Por otra parte, se trata de relacionar la proporción existente entre la dispensación sin receta y la solicitud sin receta.

\section{MÉTODOS}

El procedimiento de recogida de datos y de evaluación de los resultados se describe en la publicación correspondiente a la primera fase. ${ }^{1}$

Se ha realizado un análisis descriptivo, determinando la evolución de las variables de estudio a lo largo del tiempo, calculando y comparando porcentajes entre las fases del estudio $y$, en cada una de ellas, la solicitud y dispensación de antibióticos con y sin receta.

Como variables de estudio se contemplan: (a) farmacias participantes; (b) variables relacionadas con la solicitud; (c) variables relacionadas con la dispensación y (d) variables relacionadas con la respuesta del farmacéutico.

La unidad temporal de medida es la semana, ya que la información se ha recogido en distintas semanas de diferentes meses correspondientes a distintas estaciones del año. Se considera la medida semanal por ser el máximo nivel de desagregación disponible de los datos, y además es una definición natural (cuatro semanas de meses distintos no hacen un mes). Por otra parte, si se toma el año como medida temporal, se agregarían demasiado los datos (lo que conlleva una pérdida importante de información).

En las figuras que se presentan, hay que tener en cuenta que aunque aparecen en el eje de abscisas los nombres de los meses de estudio, en realidad los datos hacen referencia a una semana de cada uno de ellos.

\section{Análisis estadístico}

Se creó la base de datos en el programa estadístico SPSS 11.5 con la información recolectada para su posterior análisis estadístico.

Se ha puesto énfasis en las variables relacionadas con la dispensación sin receta. Para ello se ha realizado un análisis detallado de la dispensación sin receta por fases, tanto por prescripción oral del médico como del dentista, y de la actuación del farmacéutico ante una solicitud sin receta de un antibiótico.

En cuanto a la información referente a la solicitud de antibióticos, se ha considerado el $\mathrm{n}^{\circ}$ de antibióticos solicitados por farmacia y por semana. Este cálculo se ha realizado de la siguiente manera: cociente del número de antibióticos solicitados en cada semana concreta, entre el número de farmacias participantes esa semana. De esta manera, se anula la distorsión en los resultados a la que podría llevarnos el número variable de farmacias en cada uno de los períodos de estudio.

Por otro lado y referente a los valores de la dispensación, aunque se han incluido valores absolutos, parte de la información se ha presentado en porcentajes. Esta información porcentual no hace referencia al 'ajuste por farmacia', ya que, al realizar el cálculo en relación al número de farmacias, se observa que los resultados carecen del 'efecto distorsionador'.

$$
\frac{A / n^{\mathrm{o}} \text { _farmacias }}{B / n^{\mathrm{o}} \text { farmacias }}=A / B
$$

Se ha tomado como variable dependiente (o resultado) la dispensación y dentro de ella, la "dispensación de antibióticos sin receta", y como variable independiente (o predictora) las "fases". Se ha llevado a cabo una comparación de medias con el posterior análisis de varianza de un factor (ANOVA de un factor) a fin de analizar si existen diferencias estadísticamente significativas entre las variables: "dispensación de antibióticos sin receta" y "fases". 
Para medir la relación entre la "dispensación de antibióticos sin receta" y la variable independiente, se ha realizado un modelo de regresión lineal simple representando los resultados en un gráfico de dispersión.

Para conocer la relación existente entre la distinta proporción de antibióticos dispensados sin receta y solicitados sin receta, se ha llevado a cabo el análisis de regresión lineal simple y el gráfico de dispersión entre ambas variables donde se ha tomado como variable dependiente el porcentaje de dispensación sin receta y como variable independiente el porcentaje de solicitud sin receta.

Se ha relacionado cada actuación del farmacéutico (variable dependiente), con la solicitud de antibióticos sin receta como variable independiente, para analizar si existe o no una asociación estadísticamente significativa. También se ha realizado un análisis de regresión lineal simple para conocer la tendencia y el grado de asociación existente entre las variables.

Hay que tener en cuenta que en el capítulo de resultados del análisis bivariante, se han incluido únicamente aquellos resultados estadísticamente significativos, descartando el resto por carecer de interés al no existir asociación entre algunas de las variables predictoras con la variable dependiente.

\section{RESULTADOS}

La media de oficinas de farmacia (SD) existentes en Gipuzkoa durante el periodo de estudio es de $279,5(0,84)$ y la media de las que participaron enviando datos fue de 142, aproximadamente la mitad, oscilando por semanas, entre un mínimo de 106 y un máximo de 167 farmacias, habiéndose estabilizado en la última fase en un número de participantes próximo a 150.

\section{Solicitud de antibióticos}

El número total de antibióticos solicitados en el periodo de estudio fue de 83.464. De ellos, 5.890 $(7,0 \%)$ fueron demandados sin receta médica.

En la tabla 1 se indica la evolución a lo largo del programa de la solicitud total y de la solicitud sin receta, así como de los valores ajustados por oficina de farmacia (N/OF) y de la proporción de los solicitados sin receta respecto a los totales. También se muestra la evolución de la demanda sin receta, diferenciando si fueron prescritos oralmente por el médico o el dentista, o fueron solicitados por automedicación.

La Figura 1 refleja la evolución en porcentajes del origen de la solicitud sin receta: por prescripción oral y por automedicación, respecto a la solicitud de antibióticos sin receta.

\begin{tabular}{|l|c|c|c|c|c|c|c|}
\hline \multirow{2}{*}{ Tabla 1. Solicitud de antibióticos según fase del estudio y origen de la solicitud sin receta. } \\
\hline & \multicolumn{3}{|c|}{ Ab ST } & \multicolumn{2}{c|}{ Ab S-SR } & $\begin{array}{c}\text { Ab } \\
\text { S-SR/ST }\end{array}$ & $\begin{array}{c}\text { Ab S-SR } \\
\text { en función del origen }\end{array}$ \\
\cline { 2 - 9 } & $\mathrm{N}\left(\%{ }^{*}\right)$ & $\mathrm{N} / \mathrm{OF}$ & $\mathrm{N}\left(\%^{*}\right)$ & $\mathrm{N} / \mathrm{OF}$ & $\%$ & $\begin{array}{c}\text { Ab S-SR } \\
\text { por PO }\end{array}$ & $\begin{array}{c}\text { Ab S-SR } \\
\text { por AM }\end{array}$ \\
\hline Primera & $21.361(25,6)$ & 174,8 & $2.269(38,5)$ & 18,1 & 10,6 & 1.515 & 754 \\
\hline Segunda & $20.992(25,1)$ & 155,4 & $1.775(30,1)$ & 13,2 & 8,5 & 1.154 & 621 \\
\hline Tercera & $21.770(26,1)$ & 137,5 & $1.144(19,4)$ & 7,2 & 5,3 & 672 & 472 \\
\hline Cuarta & $19.341(23,2)$ & 131,0 & $702(11,8)$ & 4,8 & 3,6 & 397 & 305 \\
\hline $\begin{array}{l}\text { Total } \\
\text { periodo }\end{array}$ & $83.464(100)$ & 598,7 & $5.890(100)$ & 43,3 & & 3.738 & 2.152 \\
\hline $\begin{array}{l}\text { *Porcentaje de la fase correspondiente respecto al total del periodo de estudio } \\
\text { Ab ST: total de antibióticos solicitados; Ab S-SR: antibióticos solicitados sin receta; Ab S-SR por PO: } \\
\text { antibióticos solicitados sin receta por prescripción oral; Ab S-SR por AM: antibióticos solicitados sin receta } \\
\text { por automedicación. }\end{array}$ \\
\hline
\end{tabular}

\begin{tabular}{|c|c|c|c|c|c|}
\hline \multirow{2}{*}{ Fases } & $\mathrm{Ab} D T$ & $A b D-S R$ & \multirow{2}{*}{$\begin{array}{c}\mathrm{Ab} \\
\mathrm{D}-\mathrm{SR} / \mathrm{DT} \\
(\%)\end{array}$} & \multirow{2}{*}{$\begin{array}{c}\text { Ab D-SR } \\
\text { por PO } \\
\mathrm{N}\left(\%^{*}\right)\end{array}$} & \multirow{2}{*}{$\begin{array}{c}\text { Ab D-SR } \\
\text { por AM } \\
\mathrm{N}\left(\%^{*}\right)\end{array}$} \\
\hline & $\mathrm{N} \quad\left(\%^{*}\right)$ & $\mathrm{N} \quad\left(\%^{*}\right)$ & & & \\
\hline Primera & $20.692(25,5)$ & $(44,2)$ & 7,7 & $1.382(42,9)$ & $218(54,6)$ \\
\hline Segunda & $20.419(25,1)$ & $(33,1)$ & 5,9 & $1.079(33,5)$ & $119(29,8)$ \\
\hline Tercera & $21.151(26,0)$ & $(14,5)$ & 2,5 & $489(15,2)$ & $36(9,0)$ \\
\hline Cuarta & $18.939(23,3)$ & $(8,2)$ & 1,6 & $270(8,4)$ & $26(6,5)$ \\
\hline $\begin{array}{l}\text { Total } \\
\text { periodo }\end{array}$ & $81.201(100)$ & 3.619 (100) & & $3.220(100)$ & $399(100)$ \\
\hline \multicolumn{6}{|c|}{$\begin{array}{l}\text { *Porcentaje de la fase correspondiente respecto al total del periodo de estudio } \\
\text { Ab DT: total de antibióticos dispensados; Ab D-SR: antibióticos dispensados sin receta; Ab D-SR por PO: } \\
\text { antibióticos dispensados sin receta por prescripción oral; Ab D-SR por AM: antibióticos dispensados sin receta } \\
\text { por automedicación. }\end{array}$} \\
\hline
\end{tabular}

\section{Dispensación de antibióticos}

En cuanto a los antibióticos dispensados en el periodo analizado, el total ha ascendido a 81.201, de los cuales 3.619 han sido sin la correspondiente receta médica, como se muestra en la tabla 2.
En la figura 2 se muestra la tendencia del porcentaje de antibióticos totales dispensados respecto al total de solicitados, a lo largo del periodo de estudio. 
La figura 3 ilustra la tendencia de la dispensación con y sin receta respecto al total de antibióticos dispensados a lo largo del periodo de estudio.

En la tabla 3 se muestra la comparación de medias de antibióticos dispensados sin receta en las cuatro fases del estudio con el posterior análisis de varianza (ANOVA) para analizar la fuerza de asociación. Se refleja una disminución considerable de la media de éstos a lo largo del transcurso de cada fase, siendo esta relación estadísticamente significativa $(p<0.01)$.

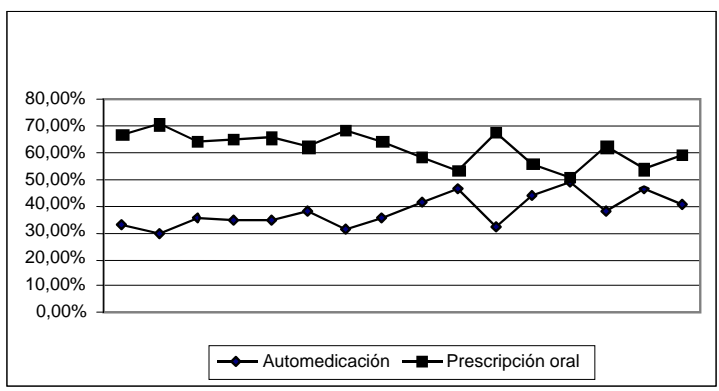

Figura 1. Antibióticos solicitados sin receta

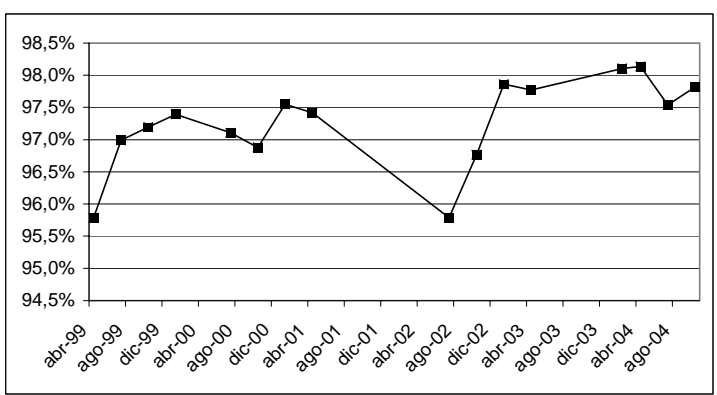

Figura 2. Porcentajes de antibióticos dispensados

Tabla 4: Solicitud de antibióticos totales y sin receta, dispensación sin receta, relación entre la dispensación sin receta y la solicitud sin receta.

\begin{tabular}{|l|c|c|c|c|c|c|}
\hline Fases & Ab ST & Ab S-SR & Ab D-SR & Ab NoD & $\begin{array}{c}\% \\
\text { NoD/S-SR }\end{array}$ & $\begin{array}{c}\% \\
\text { D-SR/S-SR }\end{array}$ \\
\hline Primera & 21.361 & 2.269 & 1.600 & 669 & 29,5 & 70,5 \\
\hline Segunda & 20.992 & 1.775 & 1.198 & 577 & 32,5 & 67,5 \\
\hline Tercera & 21.770 & 1.144 & 525 & 619 & 54,1 & 46,0 \\
\hline Cuarta & 19.341 & 702 & 296 & 406 & 57,8 & 42,2 \\
\hline $\begin{array}{l}\text { Total } \\
\text { periodo }\end{array}$ & 83.464 & 5.890 & 3.619 & 2.271 & 38,6 & 61,4 \\
\hline
\end{tabular}
sin receta; Ab NoD: antibióticos no dispensados

\begin{tabular}{|c|c|c|c|c|c|c|}
\hline \multirow[b]{2}{*}{ Fases } & \multirow[b]{2}{*}{ Ab S-SR } & \multicolumn{3}{|l|}{ No dispensó } & \multicolumn{2}{|l|}{ Dispensó } \\
\hline & & $\begin{array}{l}\text { Remitió al } \\
\text { médico }\end{array}$ & $\begin{array}{l}\text { Ofreció tratamiento } \\
\text { alternativo }\end{array}$ & $\begin{array}{l}\text { Ambas } \\
\text { acciones }\end{array}$ & $\begin{array}{l}\text { Sólo } \\
\text { Dispensó }\end{array}$ & $\begin{array}{l}\text { Además } \\
\text { remitió } \\
\text { médico }\end{array}$ \\
\hline Primera & 2.269 & 407 & 130 & 132 & 1.342 & 258 \\
\hline Segunda & 1.775 & 336 & 114 & 127 & 999 & 199 \\
\hline Tercera & 1.144 & 428 & 102 & 89 & 394 & 131 \\
\hline Cuarta & 702 & 300 & 47 & 59 & 220 & 76 \\
\hline $\begin{array}{l}\text { Total } \\
\text { periodo }\end{array}$ & 5.890 & 1.471 & 393 & 407 & 2.955 & 664 \\
\hline
\end{tabular}

El gráfico de dispersión de la figura 7, muestra visualmente la correlación lineal y positiva entre las variables de dispensación sin receta y la solicitud sin receta, en términos de porcentajes. El resultado de la regresión confirma que están relacionadas de
En la figura 4 se muestra la relación entre la dispensación de antibióticos sin receta por prescripción oral del médico o dentista y la solicitud de ellos de acuerdo al análisis de regresión lineal $(p<0,01)$.

\begin{tabular}{|c|c|c|}
\hline \multicolumn{3}{|c|}{$\begin{array}{l}\text { Tabla 3. Antibióticos dispensados sin receta y año d } \\
\text { estudio. }\end{array}$} \\
\hline Fases & Media & Desv. típica \\
\hline Primera & 396,92 & 60.383 \\
\hline Segunda & 306,26 & 47.248 \\
\hline Tercera & 129,28 & 21,324 \\
\hline Cuarta & 74,38 & 12,437 \\
\hline Total & 229,57 & 135,249 \\
\hline
\end{tabular}

En la figura 5 se muestra a través del cálculo de regresión lineal $(0,3)$, la relación entre solicitud de antibióticos a petición propia, con la dispensación por automedicación con una fuerza de asociación menor que la anterior $(p<0,01)$.

\section{Antibióticos solicitados sin receta y dispensados}

En la tabla 4 se muestra la evolución global de los antibióticos tanto solicitados como dispensados sin receta; así como los no dispensados y la relación entre ellos.

La figura 6 muestra a través de un gráfico de dispersión, como la relación existente entre el número de antibióticos solicitados y dispensados sin receta está fuertemente correlacionada linealmente y de forma positiva $(P<0,01)$ y con un coeficiente de regresión de 0,83 manera significativa $(p<0,01)$, dándose un coeficiente de regresión de 0,87. 


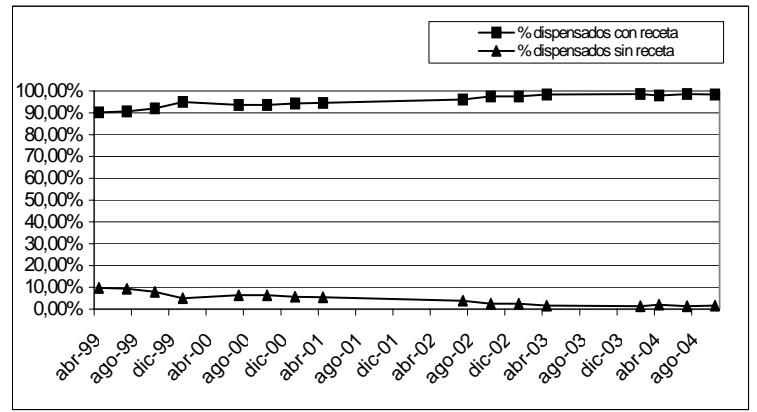

Figura 3. Porcentajes de antibióticos dispensados, con y sin receta

\section{Actuación del farmacéutico}

En la tabla 5 se muestra la actuación del farmacéutico ante la solicitud de un antibiótico sin receta a lo largo del periodo de estudio; especificando las acciones tanto en los casos en que se dispensó como en los que no lo hizo.

Para analizar la relación entre la actuación del farmacéutico con la solicitud de antibióticos sin receta, se han realizado análisis de regresión lineal para cada una de las actuaciones del farmacéutico ante la solicitud de un antibiótico sin receta. De

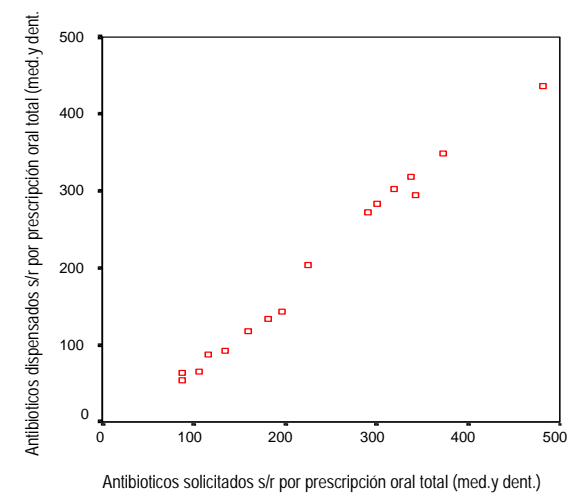

Figura 4

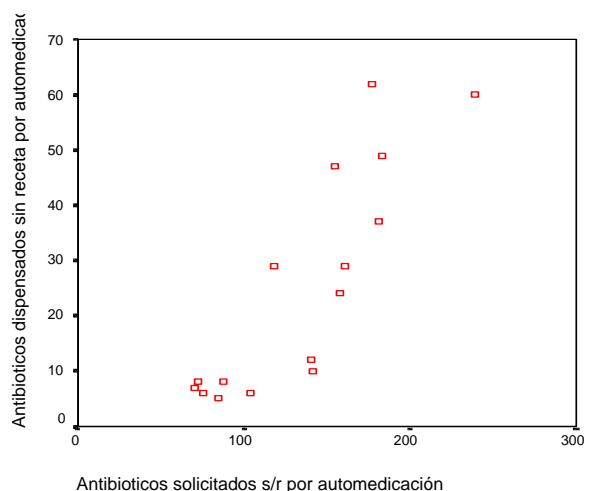

Figura 5

\section{DISCUSIÓN}

En este artículo se analizan los resultados del Programa de uso racional de antibióticos en Guipúzcoa desde el año 1999 hasta el 2004. todas ellas se destacan únicamente los resultados que tienen significación desde el punto de vista estadístico, el resto no se incluye por su escaso interés.

En la figura 8, muestra de manera visual la correlación lineal entre las variables "Farmacéutico remitió al médico y dispensó" y "antibióticos solicitados sin receta". El análisis de regresión lineal simple realizado, señala que este tipo de actuación del farmacéutico está asociado de forma significativa con la solicitud de un antibiótico sin receta $(p<0.01)$ de manera que cada año del estudio transcurrido disminuyen ambas variables, relacionadas entre sí desde el punto de vista del análisis estadístico, con un coeficiente de regresión de 0,11 .

En la figura 9, se refleja la correlación entre las variables "Farmacéutico dispensó antibiótico" y "antibióticos solicitados sin receta". El análisis de regresión lineal simple llevado a cabo señala que están asociadas de manera significativa $(p<0,01)$, dándose un coeficiente de regresión de 0,71.

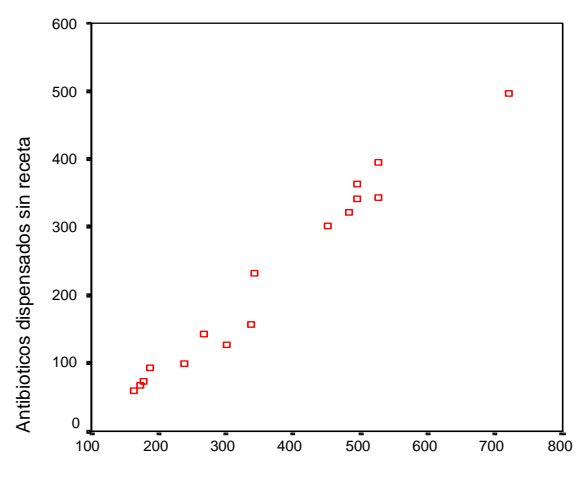

Antibioticos solicitados sin receta

Figura 6

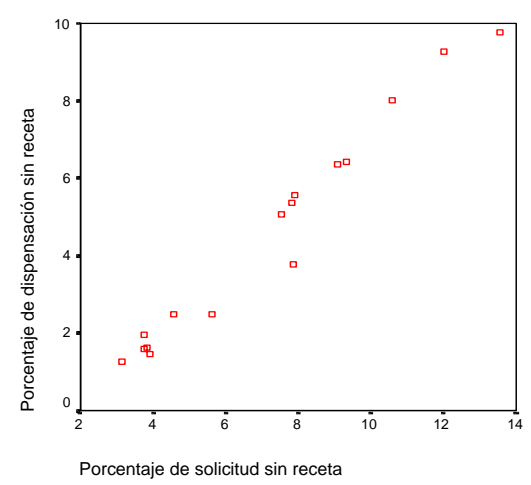

Figura 7

Tal y como se describió en nuestra publicación anterior, ${ }^{1}$ se partía de una situación social de aceptación generalizada del uso de antibióticos sin receta que era necesario modificar. Esta misma idea ha sido defendida por otros autores. ${ }^{2-5}$ Tras los resultados obtenidos hasta el momento, seguimos 
pensando que este Programa debe ser potenciado y ampliado al mayor número de farmacias posible. Se ha conseguido no solo mejorar la educación sanitaria del paciente a través de la información facilitada directamente por el farmacéutico, sino también concienciar a la sociedad a través de los medios de comunicación.

La tendencia global de solicitud total de antibióticos se mantuvo constante a lo largo de las cuatro fases del estudio (tabla 1). La solicitud total de antibióticos incluye, además de la solicitud de antibióticos sin receta, la solicitud de antibióticos con receta que es mayoritaria.

Si se tiene en cuenta la solicitud de antibióticos sin receta respecto a la solicitud total de antibióticos, la proporción de éstos bajó considerablemente desde un $10,6 \%$ en la primera fase, hasta un $3,6 \%$ en la última (tabla 1). Esto nos permite afirmar que a lo largo del periodo de estudio se ha conseguido una disminución considerable en la solicitud de antibióticos sin receta por parte de los usuarios.

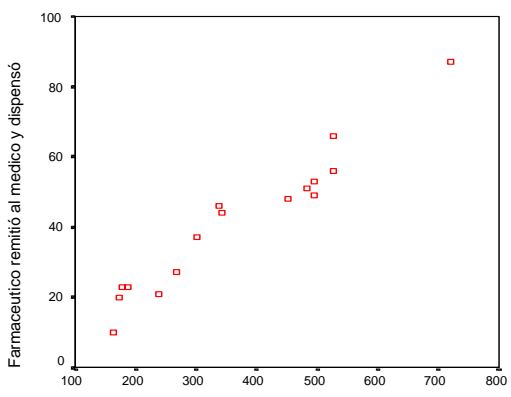

Antibioticos solicitados sin receta

Figura 8.

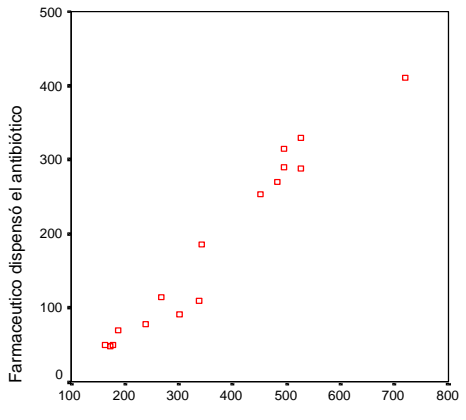

Antibioticos solicitados sin receta

Figura 9.

Este bajo porcentaje de solicitudes sin receta $(3,6 \%)$ es un dato inferior a los descritos en la literatura $\left[23 \%(\text { Salar, 2005) })^{6}\right.$ y $6 \%$ (Artetxe, $2003)^{7}$ ]. La diferencia entre nuestros resultados y el resto puede deberse a la continua campaña de educación a la población que se viene realizando desde el año 1999 en el Colegio de Farmacéuticos de Gipuzkoa, pudiéndose considerar éste como el I Programa Institucional de Uso Racional de Antibióticos en España.

La dispensación total de antibióticos también se ha mantenido constante observándose sin embargo, una disminución estadísticamente significativa en la dispensación sin receta a lo largo del estudio.
Disminuyó desde un 7,7\% en la primera fase hasta un $1,6 \%$ en la última (tabla 2). Existe una amplia variabilidad en los resultados sobre dispensación de antibióticos sin receta. [65,9\% (Caamaño, $2005)^{8}, 55,4 \%$ (Barris, 2005) $^{9}, 55 \%$ (COF Bizkaia, $2000)^{10}, 41 \%$ (Väänänen, 2005) ${ }^{11}, 32 \%$ (Orero, 1998) ${ }^{12}$ ', 31\% (Salar, 2005) ${ }^{6}$, 5\% (COF Bizkaia, $\left.2004)^{10}\right]$. En nuestra opinión, esta variabilidad es probablemente, reflejo no sólo de las distintas metodologías utilizadas en los diferentes estudios, ${ }^{13}$ sino también de la variabilidad real que existe en la actuación profesional del farmacéutico en nuestro país.

En este sentido, Barris $^{9}$ afirma que en su medio, a diferencia de lo que ocurre en otras provincias 1,10 las solicitudes de antibióticos sin receta no han sufrido modificaciones a lo largo del tiempo. Además, este autor manifiesta que sus usuarios se resisten a admitir sus intervenciones hasta en un 56 $\%$. Esto también ocurre en nuestro estudio, en el que se dispensa el $42,2 \%$ de las solicitudes sin receta (tabla 4) y en el de Salar y $\mathrm{col}^{6}$, que afirman que el $31 \%$ de los pacientes no aceptaron sus intervenciones. En nuestra opinión, estos porcentajes no hacen referencia exactamente a la misma realidad. Creemos importante matizar que en Gipuzkoa, tras cuatro años de Programa, y una importante disminución en las solicitudes de antibióticos sin receta, éstas provienen de pacientes que son menos permeables a las intervenciones del farmacéutico.

Para favorecer la mejora de la actuación del farmacéutico, Barris ${ }^{9}$ propone la implantación de programas en los que participen todos los agentes implicados (médicos, farmacéuticos, usuarios, etc.) como ocurre en nuestro caso ${ }^{1}$ y en otros. ${ }^{10} \mathrm{Al}$ igual que insiste en la necesidad de coordinación en las actuaciones institucionales y la utilización de guías de actuación para los farmacéuticos, lo que a nuestro parecer podría reforzarse con la existencia de incentivos negativos.

Podemos afirmar que, en nuestro caso, esta disminución de la dispensación sin receta fundamentalmente ha sido consecuencia de la disminución en la solicitud de antibióticos sin receta, porque ambas variables se encuentran fuertemente correlacionadas y de forma positiva tal como se muestra en la figura 6. Además, se mantiene la asociación estadísticamente significativa entre la solicitud por prescripción oral por médico o dentista y su posterior dispensación (figura 4). Ocurre lo mismo con la automedicación, aunque la fuerza de asociación es más débil que en el caso anterior (figura 5). Según estos datos, en general, el farmacéutico no se cuestiona la dispensación cuando el usuario mantiene que la prescripción es telefónica o mediada por una recomendación anterior del médico.

En esta discusión nos interesa mucho analizar la respuesta del farmacéutico ante una solicitud de un antibiótico sin la receta correspondiente, ya que uno de los objetivos del Programa es mejorar precisamente dicha actuación. 
La respuesta del farmacéutico ante la solicitud de antibióticos sin receta fue diversa. El farmacéutico, en la última fase del estudio, no dispensó el antibiótico en el $57,8 \%$ (tablas 4 y 5 ) cifra algo inferior a la obtenida por Salar y col $^{6}$ que es de un $69 \%$. Cuando no dispensó, el farmacéutico: (a) Remitió el paciente al médico, (b) ofreció un tratamiento alternativo, ó (c) realizó ambas acciones. Cuando dispensó el antibiótico, en algunas ocasiones además remitió el paciente al médico.

En este trabajo se ha realizado un análisis de regresión lineal para cada una de las actuaciones del farmacéutico ante la solicitud de un antibiótico sin receta. De todas ellas a continuación discutimos únicamente aquellos resultados que tienen significación desde el punto de vista estadístico. El resto no se incluyen por su escaso interés.

Aunque el farmacéutico pasó de dispensar sin receta desde un $70,5 \%$ de las solicitudes que le llegaron en la primera fase, a un $42,2 \%$ en la última fase del Programa (tabla 4), esto no se debe entender como un cambio sustancial en su actuación profesional, sino que principalmente, es debido a la disminución de las solicitudes sin receta. De hecho, el análisis de regresión lineal simple llevado a cabo, señala que entre las variables "farmacéutico dispensó el antibiótico" y "antibióticos solicitados sin receta", están asociadas de manera significativa (figura 9). Esta disminución de solicitudes, pensamos que es consecuencia de que el paciente ha sido receptivo a la actuación colectiva de todos los agentes implicados en la utilización del medicamento.

Además, sí que podemos afirmar que el farmacéutico cada vez remite más al médico cuando realiza una dispensación sin la correspondiente receta $(11,3 \%)$, con una relación significativa (figura 8).

Es evidente que queda todavía espacio de trabajo para conseguir que la dispensación sin receta se convierta en algo casi testimonial, reservado tan sólo a casos de urgencia y necesidad. Sin embargo, se debería profundizar en algún sistema para que en aquellos casos de necesidad, en que el farmacéutico con la aceptación del paciente y siguiendo los protocolos o las directrices de las Guías de Práctica Clínica, pueda tomar una decisión terapéutica con el compromiso de ponerlo posteriormente en conocimiento del médico. De la misma manera, se deberían articular procedimientos para aquellos casos excepcionales en los que el médico u odonto-estomatólogo necesita realizar una prescripción oral de un antibiótico. ${ }^{14}$

No podemos finalizar esta discusión sin hacer una breve reflexión sobre la relación entre las resistencias bacterianas y la dispensación de antibióticos sin receta. Se responsabiliza con mucha frecuencia al farmacéutico de ser copartícipe en los efectos negativos asociados al uso inapropiado de los antibióticos ${ }^{15}$ incluso se culpa a estos profesionales de contribuir decisivamente a la expansión y exportación de estas resistencias. ${ }^{11}$

Como se observa en la figura 3 , al comenzar el estudio, aproximadamente el $90 \%$ de los antibióticos se dispensaban con receta superando el $98 \%$ al finalizar el mismo. Esto supone que, al menos en nuestro entorno, el uso inadecuado del antibiótico no puede ser achacable principalmente al farmacéutico. Estamos completamente de acuerdo en la necesidad de mejorar el cumplimiento de la legislación vigente en relación a la exigencia de receta médica, pero las consecuencias del uso inapropiado de los antibióticos (resistencias, etc.) habrá que buscarlas también en otros ámbitos o factores. ${ }^{16}$

La participación en el Programa es voluntaria por lo que no responden todas las farmacias y las que lo hacen, no son siempre las mismas. Esto hace que se desconozca lo que ocurre en las farmacias que no han contestado nunca a las encuestas, aunque es preciso reseñar que la participación es muy elevada (aproximadamente el 50\%).

\section{CONCLUSION}

Los resultados que está obteniendo este I Programa Institucional de Uso Racional de Antibióticos en Gipuzkoa están siendo, en términos generales, satisfactorios.

Se ha conseguido una disminución muy importante en el número de personas que acuden a la farmacia a solicitar un antibiótico sin la correspondiente receta médica.

Como consecuencia de ese descenso de solicitudes, ha disminuido de manera estadísticamente significativa la dispensación de antibióticos sin receta en las farmacias guipuzcoanas.

En los casos en los que dispensa sin receta, cada vez remite más pacientes al médico una vez procedida la dispensación.

Sin embargo, el farmacéutico no ha modificado sustancialmente su actuación profesional, por lo que es necesario seguir trabajando en reforzar su actuación como agente sanitario. Para ello proponemos la implicación más activa de las instituciones, la utilización de guías de práctica clínica por parte de los profesionales y la posible aplicación de incentivos negativos.

A nuestro juicio, el éxito de este Programa radica en la actuación coordinada de todos los agentes implicados en la cadena del uso del antibiótico.

Dados los resultados obtenidos, nos atrevemos a afirmar que este tipo de actuaciones coordinadas con otros profesionales sanitarios son la clave del éxito, sin olvidar la importancia de la difusión a los usuarios.

\section{AGRADECIMIENTOS}

A los farmacéuticos guipuzcoanos que colaboran enviando sus datos para la elaboración de este 
trabajo. A la Junta de Gobierno y Secretario Técnico del Colegio Oficial de Farmacéuticos de Gipuzkoa por su estímulo y apoyo económico, así como a su personal administrativo. A Cecilia Escudero y a Joe Ling Chang por el análisis estadístico de los datos.

\section{Referencias}

1 Gastelurrutia MA, Larrañaga B, Ortega B, Puntonet L. Evaluación del programa de uso racional de antibióticos en Gipuzkoa. Primera fase: 1999-2000. Pharm Care Esp 2002; 4: 143-57.

2 Red Española de Atención Primaria. Los antibióticos, con receta. Documento de Valencia. Pharm Care Esp 2000; 2:2013.

3 Ripoll MA. Con los antibióticos no se juega. Medicina General 2002; 42: 167-8.

4 Gérvas J. La resistencia a los antibióticos, un problema de salud pública. Aten Primaria 2000; 25: 589-96.

5 Candel FJ, Matesanz M, Candel I, Betriu C, Picazo JJ. ¿Qué hacer frente al aumento de la resitencia a los antibióticos?. JANO 2003; 64 (1462): 35-40.

6 Salar L. Primer Programa Nacional de Atención Farmacéutica sobre Uso Racional de Antibióticos. Pharm Care Esp 2005; 7: 56-58.

7 Artetxe E. Estudio de utilización de antibióticos en una farmacia comunitaria. Pharm Care Esp 2003; 5: 253-260.

8 Caamaño F, Tomé-Otero M, Takkouche B, Gestal-Otero JJ. Influence of pharmacists'opinions on their dispensing medicines without requirement of a doctor's prescription. Gac Sanit 2005; 19 (1): 9-14.

9 Barris D, Rodríguez Zarzuelo C, Sabio B, Garrido B, Gutiérrez Alvárez JL, Martínez-Rey A. Evolución de la demanda de antibióticos orales sin receta en una farmacia comunitaria. Seguimiento Farmacoterapéutico 2005; 3(2): 84-9.

10 Anónimo. Drástico descenso del número de farmacias vizcainas que dispensan antibióticos sin receta. Offarm 2004; 23(7): 29.

11 Cabiedes L. La punta del iceberg: venta de antibióticos sin receta....a fineses residentes en España. Gestión Clínica y Sanitaria 2005; 7(4): 153.

12 Orero A, Ripoll MA, González J. Análisis de la automedicación con antibióticos en la población española. Enferm Infecc Microbiol Clin 1998; 16-328-33.

13 Pastor Sánchez R. Problemas con los antibióticos. Aula de la farmacia 2004; 1(2): 52-9.

14 Saint S, Scholes D, Finn SD, Farell RG, Stamm WE. The effectiveness of a clinical practice guideline for the management of presumed uncomplicated urinary tract infection in women. Am J Med 1999; 106(6): 636-41.

15 Palop V, Melchor A, Martinez Mir I. Reflexiones sobre la utilización de antibióticos en atención primaria. Aten Primaria 2003; 32(1): 42-7.

16 Finch RG, Metlay JP, Davey PG, Baker LJ. Educational interventions to improve antibiotic use in the community: report from the International Forum on Antibiotic Resistance (IFAR) colloquium, 2002. Lancet Infect Dis 2004; 4: 44-53. 